\title{
Steigerung des Autonomiegrades von autonomen Transportrobotern im Bereich der Intralogistik - technische Entwicklungen und Implikationen für die Arbeitswelt 4.0
}

\author{
Joachim Tödter, Volker Viereck, Tino Krüger-Basjmeleh und \\ Thomas Wittmann
}

\section{Einleitung}

Das Konzept der ,Industrie 4.0` (Promotorengruppe 2013) basiert auf neuen, Mechanik, Elektronik und Informatik integrierenden Technologien für hauptsächlich industrielle Anwendungen. Neben der Produktionstechnik im engeren Sinne ist die Produktionslogistik oder genereller: die Intralogistik - eines der zentralen Anwendungsfelder.

In diesem Beitrag werden zunächst aktuelle technische Entwicklungen aus der Perspektive von STILL dargestellt. Es folgen einige Überlegungen zu möglichen Folgen dieser Entwicklungen für Unternehmen und Arbeitswelt.

STILL bietet maßgefertigte innerbetriebliche Logistiklösungen weltweit und realisiert das intelligente Zusammenspiel von Gabelstaplern und Lagertechnik, Software, Dienstleistungen und Service. Mit über 7.000 Mitarbeitern, vier Produktionsstätten, 14 Niederlassungen in Deutschland, 20 Tochtergesellschaften im Ausland, sowie einem weltweiten Händlernetz ist STILL erfolgreich international tätig. Mit höchster Qualität, Zuverlässigkeit und innovativer Technik, erfüllt STILL heute und in Zukunft die Anforderungen kleiner, mittlerer und großer Unternehmen.

\section{Herausforderung des Marktes}

Aufgrund der gestiegenen Komplexität und Dynamik der Einsatzgebiete spielen Flexibilität und Wandelbarkeit in der Logistik eine immer wichtigere Rolle. Darüber hinaus nimmt auch die Bedeutung von Automatisierungen der innerbetrieblichen Logistikprozesse rasant

Der Originaltext dieses Beitrags wurde überarbeitet. Das vollständige Korrekturverzeichnis finden Sie am Ende des Buchs und online unter http://dx.doi.org/10.1007/978-3-662-45915-7_16.

J. Tödter · V. Viereck (凶) · T. Krüger-Basjmeleh · T. Wittmann

Vorentwicklung/Advance Development (EVE), STILL GmbH, Berzeliusstraße 10,

22113 Hamburg, Deutschland

e-mail: Volker.Viereck@still.de

(C) The Author(s) 2015

A. Botthof, E.A. Hartmann (Hrsg.), Zukunft der Arbeit in Industrie 4.0,

DOI 10.1007/978-3-662-45915-7_8 
zu. Bedingt durch die kontinuierlich wachsenden Anforderungen rückt die Teil- oder Vollautomatisierung von diversen Lagervorgängen oder der internen Produktionsversorgung immer mehr in den Fokus. Ein Ende dieses Trends ist nicht abzusehen. Gerade kleine und mittelständische Unternehmen interessieren sich mehr und mehr für die Automatisierung von Lager- bzw. Transportabläufen.

Eine hohe Komplexität hinsichtlich der Planung und Auslegung, Ersteinrichtung und Anpassung heutiger Automatisierungslösungen erfordert jedoch jeweils den Einsatz von Spezialisten, was zu hohen und schwer kalkulierbaren Kosten für Beschaffung, Wartung und Anpassung führt, so dass die Implementierung einer solchen Automatisierungslösung dann oft nicht stattfindet.

\section{Lösungsansatz}

Um die notwendige Verringerung der Komplexität und damit eine Verringerung der Kosten für Ersteinrichtung und Anpassung einer Automatisierungslösung zu erreichen, entwickelt STILL seit einiger Zeit Automatisierungslösungen, die es dem Anwender erlauben, fahrerlose Transportsysteme selbstständig in Betrieb zu nehmen, zu betreuen und an Veränderungen der Logistikprozesse anzupassen. Richtungweisend für die Entwicklung ist dabei die Beachtung folgender Eckpunkte:

- Erhebliche Vereinfachung der Nutzung

- Deutliche Senkung von Konfigurationsaufwand und -komplexität

- Spürbare Erhöhung der Eigenintelligenz der Transportfahrzeuge

- Erhebliche Steigerung des Autonomiegrades der Fahrzeuge zur eigenständigen Anpassung der Fahrzeugreaktionen an Umgebungsveränderungen

Das folgende Kapitel zeigt im Rahmen des Forschungsprojekts „marion“1 entwickelte autonome STILL-Transportroboter, deren Fähigkeiten insbesondere in Bereich der Umgebungswahrnehmung und entsprechender eigenständiger Verhaltensanpassung liegen.

\section{STILL Forschungsaktivitäten im Bereich Robotik und Automatisierung}

Mit dem Ziel, weitreichende zukunftsorientierte Lösungen für die genannten Eckpunkte zu entwickeln, engagiert sich STILL seit einigen Jahren im Bereich der Forschung zu mobiler Robotik und arbeitet hier intensiv mit verschiedenen Instituten und Universitäten zusammen.

Beispielhaft sei an dieser Stelle das Forschungsprojekt marion - „Mobile autonome, kooperative Roboter in komplexen Wertschöpfungsketten“ - genannt.

\footnotetext{
${ }^{1}$ http://www.projekt-marion.de.
} 


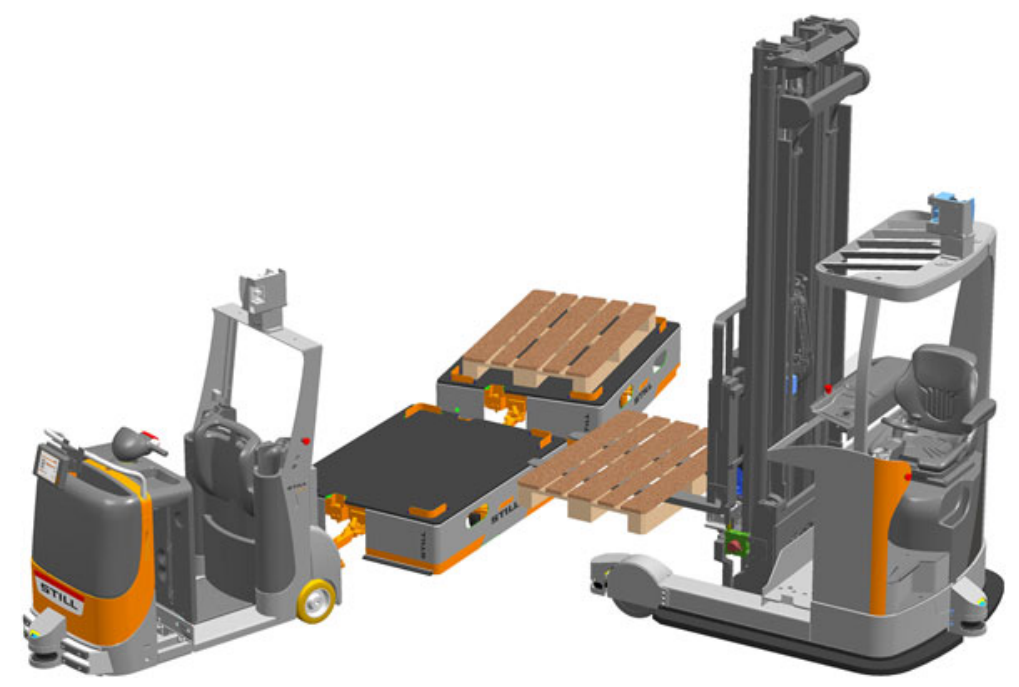

Abb. 1 Zielszenario: STILL CX-T autonom und STILL FM-X autonom kooperieren für eine vollautonome Be- und Entladung von Routenzugtrailern, Quelle: STILL GmbH

Dieses vom Bundesministerium für Wirtschaft und Energie (BMWi) geförderte Verbundprojekt hat die Automatisierung der Arbeitsprozesse mit autonomen Fahrzeugen unter besonderer Berücksichtigung der Kooperation der beteiligten Maschinen zum Ziel. Marion wird unter Beteiligung der Partner CLAAS, Atos, DFKI und STILL vom 01.08.2010 bis 30.11.2013 durchgeführt.

Die im Projekt marion im Anwendungsfall Intralogistik erzielten Ergebnisse sollen im Folgenden anhand einer beispielhaft realisierten vollautomatischen Be- und Entladung von Routenzügen verdeutlicht werden, die sowohl bei automatisierten, wie auch manuell geführten Routenzügen zur Anwendung kommen kann (Abb. 1).

Das entwickelte System ermöglicht einen flexiblen Einsatz von Transportrobotern auch in Prozessen, die einem ständigen Wandel unterliegen. Hierfür wurde ein grafisches Konfigurationstool entwickelt, das den Nutzer befähigt, einzusetzende autonome Transportroboter mit geringstem Aufwand selbst in Betrieb zu nehmen oder deren Einsatz nach Bedarf anzupassen (Abb. 2).

Dabei beschränken sich die vorzugebenden Informationen im Wesentlichen auf Fahrwege und Interaktionsbereiche, die der Nutzer für die Nutzung durch die Transportroboter freigeben möchte. Kurvenfahrten oder Manöver bspw. zur Anfahrt von Palettenstellplätzen werden dabei von den Robotern selbst berechnet und müssen nicht definiert werden.

Das dazu im marion-Projekt entwickelte dynamische Planungssystem ermöglicht es, unter den aktuellen Platzverhältnissen und Umgebungsbedingungen entsprechend optimale Fahrwege zu bestimmen, die anschließend von den Transportrobotern abgefahren werden können und eine gleichbleibend hohe Transportleistung gewährleisten. Für eine breite Akzeptanz von autonomen Fahrzeugen, die im gemeinsamen Arbeitsbereich mit dem Menschen eingesetzt werden, ist es zudem unerlässlich, dass die Fahrbewegungen 


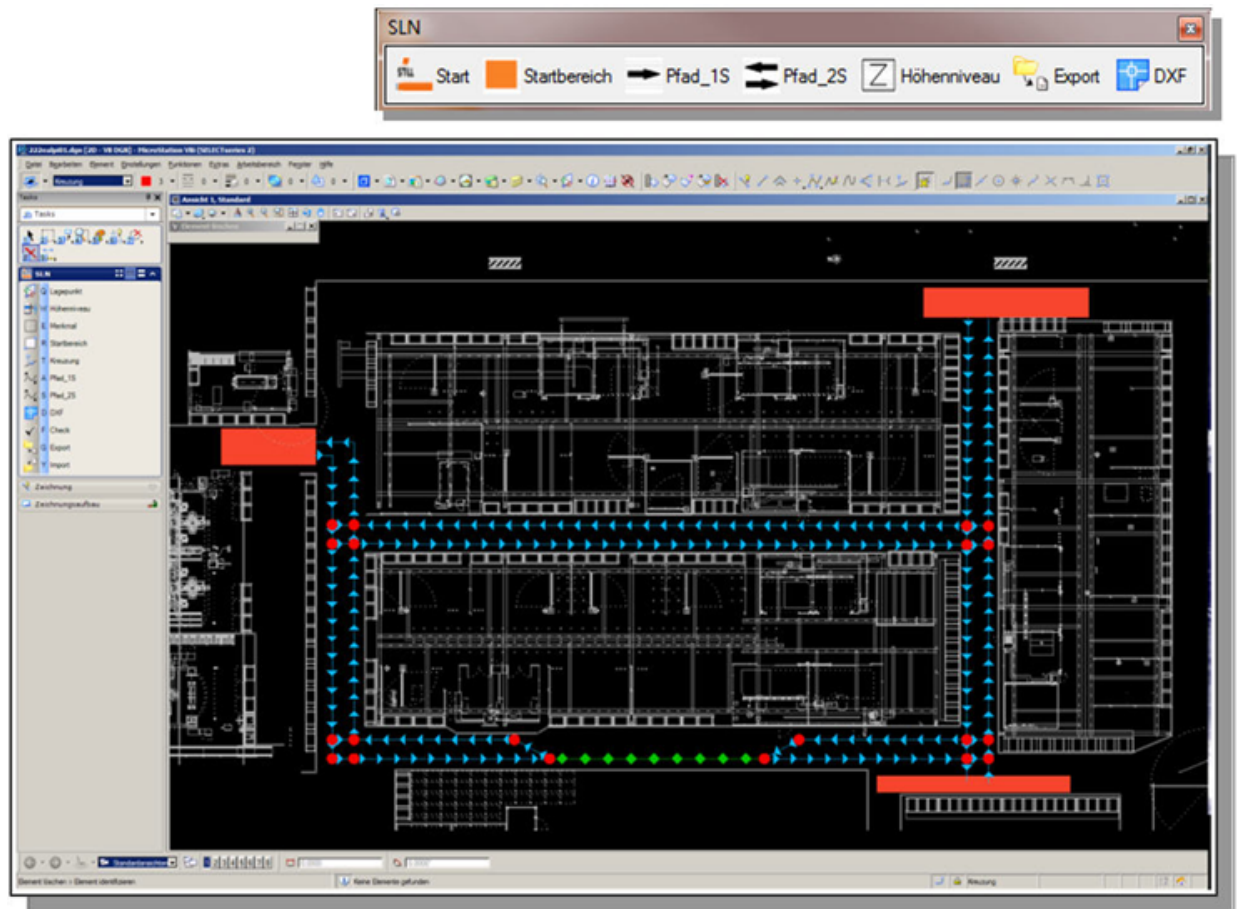

Abb. 2 Konfigurationstool zur intuitiven Einrichtung des Arbeitsbereichs der autonomen Transportroboter, Quelle: STILL GmbH

der Transportroboter zusätzlich ästhetischen Anforderungen genügen, so dass der Mitarbeiter vor Ort Vertrauen in die mit ihm eingesetzten Robotersysteme gewinnt.

Im Szenario der vollautonome Be- und Entladung von Routenzügen kommen zwei autonome STILL-Fahrzeuge zum Einsatz. Dabei handelt es sich um das Schleppfahrzeug „STILL CX-T autonom“, das mit mehreren Anhängern ausgestattet im Routenbetrieb läuft, und den autonomen Schubmaststapler „STILL FM-X autonom“, der für die Beund Entladung der Schleppzuganhänger eingesetzt wird. Die Fahrzeuge orientieren sich mit Hilfe eines 3D-Laserscanners an ihrer natürlichen Umgebung und benötigen so keine künstlichen Landmarken zur Navigation. Sie kommunizieren auf direktem Weg miteinander, tauschen benötigte Informationen aus oder delegieren Subaufträge an ein anderes Fahrzeug. Diese Funktionalität nutzt das Schleppzugfahrzeug, wenn es etwa den Auftrag bekommt, einen Ladungsträger zu einem konkreten Zielort zu transportieren. Es erfragt dazu in der Fahrzeugflotte geeignete zur Verfügung stehende Fahrzeuge und vergibt anschließend einen entsprechenden Subauftrag an das am besten geeignete Fahrzeug.

Schon während der Fahrt zum Entladeort vermisst und überwacht der Schleppzug „CXT-autonom“ per Szenenanalyse die Position seiner Anhänger und publiziert diese auch dem Entladefahrzeug „FM-X autonom“. Sobald beide Fahrzeuge den Zielort erreicht haben, plant das Entladefahrzeug einen kostenoptimalen Fahrweg zum zu entladenden An- 


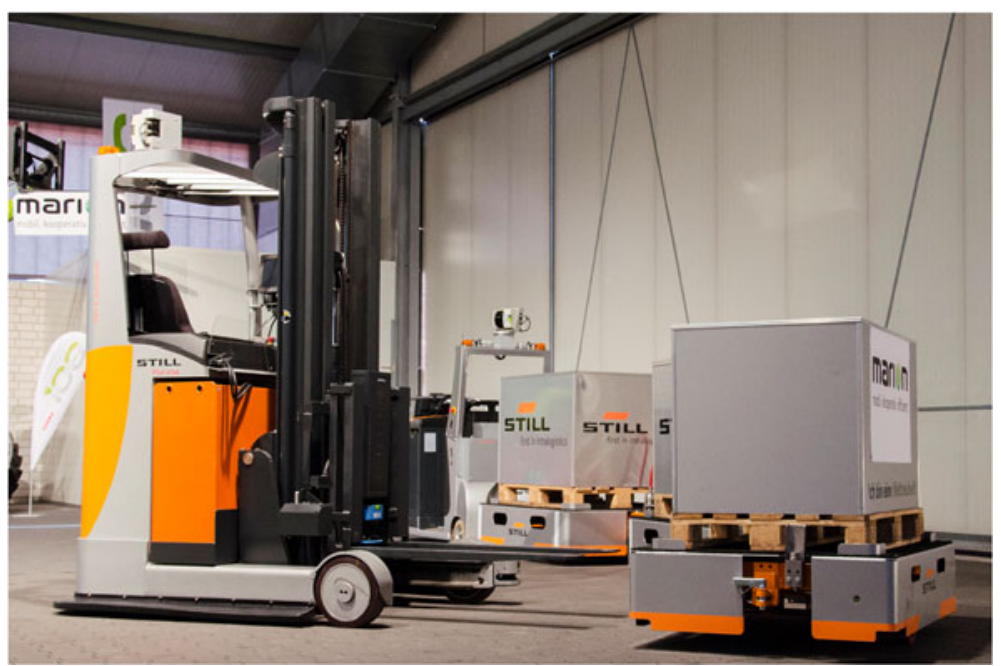

Abb. 3 Umsetzung einer selbstorganisierten multi-roboter-Kooperation; Hier Abschlussmeilenstein Forschungsprojekt „marion“, Quelle: STILL GmbH

hänger und berücksichtigt dabei auch die aktuelle in 2D und 3D erfasste Umgebungs- und Hindernissituation. Der Trailer wird entladen, wobei sich das Entladefahrzeug permanent an seinem Ziel, dem Trailer bzw. der aufzunehmenden Palette, orientiert und so in der Lage ist, bestehende Resttoleranzen auszugleichen.

Wurde die Palette entladen, gehen beide Fahrzeuge erhaltenen Folgeaufträgen nach; sofern erforderlich, werden sie erneut zur Erfüllung ihnen gestellter Aufgaben eigenständig in Kooperation treten (Abb. 3).

\section{Mögliche Konsequenzen für die Arbeitswelt}

Die oben beschriebenen Entwicklungen werden die Intralogistik erheblich verändern. Dies wird auch Auswirkungen auf die Arbeitswelt haben, im Hinblick auf Beschäftigung, Arbeitsbedingungen und Qualifikationsanforderungen.

Autonom fahrende Flurförderzeuge - Transportroboter - haben zunächst wie alle Automatisierungstechnologien unmittelbare Auswirkungen auf die Beschäftigten, deren Arbeitstätigkeiten mit zunehmendem Maß auch durch automatisierte Systeme erbracht werden können.

Einer daraus resultierenden möglichen Reduzierung des Bedarfs an Fahrern für manuell gesteuerte Transportfahrzeuge (,Einfacharbeit“ - Hirsch-Kreinsen et al. 2012) steht ein zunehmender Bedarf an entsprechend höher qualifizierten Mitarbeitern gegenüber, die in der Lage sind, derartige Systeme einzurichten, zu betreuen und zu warten. Es ergeben sich folglich eher anspruchsvolle Qualifikationsanforderungen. 
Ein naheliegender und wünschenswerter Ansatz würde darin bestehen, Mitarbeiter der Intralogistik für neue, anspruchsvolle Aufgaben weiterzubilden. Dies setzt allerdings ein erhebliches Umdenken in den Unternehmen voraus: Geringqualifizierte gehören traditionell zu den Beschäftigtengruppen, die von betrieblicher Weiterbildung am wenigsten erreicht werden. Hier sind auch neue Wege der Unterstützung gerade von kleinen und mittleren Betrieben in der Weiterbildung ihrer Beschäftigten gefragt (z. B. Jäger und Kohl 2009).

Daher ist es wichtig, durch die Gestaltung der technischen Systeme darauf hinzuwirken, dass die Qualifizierungsbedarfe auf das Notwendige beschränkt bleiben. Hier spielt die intuitive Gestaltung von Mensch-Maschine-Schnittstellen, wie sie oben am Beispiel des Projekts marion beschrieben wurde, eine zentrale Rolle.

Wie die Arbeitsbedingungen und die Qualifikationsbedarfe in der Intralogistik letztlich aussehen werden, hängt von betrieblichen Organisationsparadigmen ab. ${ }^{2}$ So ist es etwa denkbar, dass Logistikaufgaben kombiniert werden mit Aufgaben im Bereich Wartung und Instandhaltung. Auch eine Integration der Logistikaufgaben - teilweise oder vollständig - in Produktionsteams ist möglich. Je nach einzelbetrieblicher Umsetzung solcher organisationsbezogenen Entscheidungen entstehen ganz unterschiedliche Profile hinsichtlich Arbeitsbedingungen und Qualifikationsbedarf.

Im Hinblick auf zukünftige Entwicklungen im Kontext des demografischen Wandels sei schließlich darauf hingewiesen, dass die oben besprochenen Rationalisierungseffekte im Sinne einer Ersetzung menschlicher Arbeitskraft - auch aus gesellschaftlicher Perspektive eine durchaus positive Bedeutung haben können: Rationalisierung hilft dabei, auch in Zeiten schlechter Verfügbarkeit von Erwerbspersonen wettbewerbsfähig produzieren und Waren umschlagen zu können.

Auch generell sind Chancen durch zunehmende Automatisierung der Logistik zu erkennen. Große Logistikunternehmen müssen u.a. aufgrund hoher Immobilienkosten bereits häufig in weniger dicht besiedelte Regionen ausweichen. Dies bringt oftmals das Problem mit sich, nicht die benötigte Qualität und Quantität von Mitarbeitern finden zu können, so dass attraktive Automatisierungslösungen dringend benötigt werden.

\section{Fazit und Ausblick}

Es bestehen Bedarfe nach einer weiteren Steigerung des Automatisierungsgrades in der Logistik, dies betrifft insbesondere den Mittelstand.

Vor diesem Hintergrund ist es wichtig, dass Automatisierungslösungen so flexibel werden, dass der Betreiber selbst sie vollständig beherrschen, einrichten und an Veränderungen anpassen kann.

Neben der hier beschriebenen weitgehenden Automatisierung durch Transportroboter nimmt auch der Bedarf nach Assistenzfunktionen für manuell betriebene Transportfahrzeuge zu. Wichtige Ziele sind dabei Fahrerentlastung und Performancesteigerung.

\footnotetext{
${ }^{2}$ Mehr dazu in den Beiträgen von Ernst Hartmann und Bernd Kärcher in diesem Band.
} 
Chancen unterstützender Assistenzsysteme für manuell betriebene Transportfahrzeuge bestehen darin, dass auch ungeübte oder geringfügig eingearbeitete Mitarbeiter hohe Performancewerte erreichen und die Mitarbeiter sich auf ihre Haupttätigkeiten konzentrieren können. Weiterhin wird die körperliche Beanspruchung reduziert, was den Anforderungen des demografischen Wandels gerecht wird.

Darüber hinaus gehende autonome Fähigkeiten der Transportfahrzeuge, wie sie in diesem Beitrag am Beispiel des Projekts marion dargestellt wurden, bieten weitere Chancen. So lösen etwa die Transportfahrzeuge ihnen gestellte Aufgaben bei einer drastisch reduzierten Menge an Konfigurationsdaten; fehlende Konfigurationsinformationen werden durch intelligente Algorithmen auf den Fahrzeugen selbst ausgeglichen. Aufwand und Kosten der Inbetriebnahme und notwendiger Anpassungen an Prozessveränderungen werden minimiert. Es ist kein externes Experten-Knowhow zur Inbetriebnahme mehr notwendig; die Systeme sind durch den Anwender vollständig beherrschbar, womit eine stärkere Unabhängigkeit des Anwenders vom Hersteller einhergeht.

Herausforderungen stellen sich im Hinblick auf zukünftige Beschäftigungsmöglichkeiten derjenigen Mitarbeiter, deren bisherige Arbeitstätigkeiten durch automatisierte Systeme ersetzt werden. Hier kommt der Weiterbildung und dem lebenslangen Lernen besondere Bedeutung zu. Gerade kleine und mittlere Unternehmen benötigen für die Bewältigung dieser Herausforderungen auch externe Unterstützung.

Die demographische Entwicklung einbeziehend kann - auf der anderen Seite - eine hochflexible Automatisierung vielfältiger Warenumschlagprozesse als Chance oder sogar als entscheidende Voraussetzung für die notwendige Veränderung heutiger Prozesse betrachtet werden.

Open Access This chapter is distributed under the terms of the Creative Commons Attribution Noncommercial License, which permits any noncommercial use, distribution, and reproduction in any medium, provided the original author(s) and source are credited.

\section{Literaturverzeichnis}

Hirsch-Kreinsen, H., Ittermann, P., \& Abel, J. (2012). Industrielle Einfacharbeit: Kern eines sektoralen Produktions- und Arbeitssystems. Industrielle Beziehungen, 19(2), 187-210.

Jäger, A., \& Kohl, M. (2009). Qualifizierung An- und Ungelernter - Ergebnisse einer explorativen Analyse zum aktuellen betrieblichen Bedarf, zukünftigen Qualifikationsanforderungen und Präventionsansätzen der Bundesagentur für Arbeit, bwp@ Berufs- und Wirtschaftspädagogik - online, Profil 2 - Akzentsetzungen in der Berufs- und Wirtschaftspädagogik, online: http://www.bwpat.de/profil2/jaeger_kohl_profil2.shtml.

Promotorengruppe Kommunikation der Forschungsunion Wirtschaft - Wissenschaft (Hrsg.) (2013). Deutschlands Zukunft als Produktionsstandort sichern - Umsetzungsempfehlungen für das Zukunftsprojekt Industrie 4.0. 